\title{
Distribution of a Ty3/gypsy-like retroelement on the A and B-chromosomes of Cestrum strigilatum Ruiz \& Pav. and Cestrum intermedium Sendtn. (Solanaceae)
}

\author{
Jéferson Nunes Fregonezi, Laurival A. Vilas-Boas, Maria Helena Pelegrinelli Fungaro, \\ Marcos Letaif Gaeta and André Luís Laforga Vanzela \\ Departamento de Biologia Geral, Centro de Ciências Biológicas, Universidade Estadual de Londrina, \\ Londrina, PR, Brazil.
}

\begin{abstract}
Retroelements are a diversified fraction of eukaryotic genomes, with the Ty1/copia and Ty3/gypsy groups being very common in a large number of plant genomes. We isolated an internal segment of the Ty3/gypsy retroelement of Cestrum strigilatum (Solanaceae) using PCR amplification with degenerate primers for a conserved region of reverse transcriptase. The isolated segment ( $\mathrm{pCs} 12$ ) was sequenced and showed similarity with Ty3/gypsy retroelements of monocotyledons and dicotyledons. This segment was used as probe in chromosomes of $C$. strigilatum and Cestrum intermedium. Diffuse hybridization signals were observed along the chromosomes and more accentuated terminal signals in some chromosome pairs, always associated with nucleolus organizer regions (NORs). The physical relationship between the hybridization sites of $\mathrm{pCs} 12$ and $\mathrm{p} T \mathrm{~T} 71$ ribosomal probes was assessed after sequential fluorescence in situ hybridization (FISH). Hybridization signals were also detected in the B chromosomes of these species, indicating an entail among the chromosomes of A complement and B-chromosomes.
\end{abstract}

Key words: Cestrum strigilatum, Cestrum intermedium, karyotype organization,

retroelements, Solanaceae, Ty3-gypsy.

Received: May 15, 2006; Accepted: November 23, 2006.

\section{Introduction}

Retroelements comprise a common group of repetitive DNA elements which are found dispersed in a number of plant genomes. Such elements form distinct subgroups and can occupy a great portion of the genome. Maize (Zea mays L.) is a very illustrative genus, since the genomes of some species can consist of $50-85 \%$ of retroelements (Brandes et al., 1997, Kumar and Bennetzen, 1999). The Ty3/gypsy and Ty1/copia retrotransposons are typical groups with long terminal repeat (LTR), abundant in the genomes of gymnosperms and angiosperms (Van Sluys et $a l ., 2001)$. These two retrotransposons differ in the gene positioning of reverse transcriptase, integrase and RNase-H, in the Pol sequence (Kumar and Bennetzen, 1999). The Ty1/copia elements occur dispersed along the chromosomes, and can vary in repeat number according to the genome (Heslop-Harrison et al., 1997). Although the Ty3/

Send correspondence to André Luís Laforga Vanzela. Laboratório de Biodiversidade e Restauração de Ecossistemas, Departamento de Biologia Geral, Centro de Ciências Biológicas, Universidade Estadual de Londrina, 86051-990 Londrina, PR, Brazil. E-mail: andrevanzela@uel.br. gypsy are less studied than Ty1/copia, they have been shown also to occur dispersed in the genome (L'Homme et al., 2000). In some species of Helianthus (Asteraceae) and some monocotyledons Ty3/gypsy-like retroelements exhibit a dispersed pattern, but they can appear concentrated in all centromeric regions (Santini et al., 2002, Kumar and Bennetzen, 1999). Additionally, Belyayev et al. (2001) found Ty3/gypsy to be associated with secondary constrictions and with subterminal and intercalary heterochromatin in both Hordeum spontaneum and Aegilops speltoides.

The Ty3/gypsy-like and other retrotransposons have been characterized in some Solanaceae representatives, such as Solanum tuberosum and Lycopersicum esculetum (Van Sluys et al., 2001), but their physical localization has not been explored.

The genus Cestrum contains sub-tree and tree species, native to tropical and subtropical America (D'Arcy, 1979). The species possess karyotypes composed of $2 \mathrm{n}=16$, with a predominance of meta- and submetacentric chromosomes (Berg and Greilhuber, 1992, 1993a,1993b). The Cestrum group is an interesting plant group because it possesses genomes with different heterochromatin families, such as C-Giemsa bands, GC-rich bands associated 
with nucleolar organizing regions (NORs), GC-rich bands not NOR-associated, AT-rich bands associated with cold sensitive regions (CSRs), C-Giemsa/CSR/AT bands and C-GC/AT neutral bands (see Berg and Greilhuber, 1992, 1993a, 1993b, Fregonezi et al., 2004, Fregonezi et al., 2006).

The objectives of this study were isolate and characterize sequences of the Ty3/gypsy group of the Cestrum strigilatum genome, and to locate physically these elements in C. strigilatum and Cestrum intermedium chromosomes because both species present B-chromosomes. The chromosome localization of Ty3/gypsy-like retroelements could help to understand the role and the dynamics of repetitive DNA in the genome and karyotype of Cestrum, as well as their relationships with the B-chromosomes of both species.

\section{Materials and Methods}

Seeds of $C$. strigilatum were collected at São Jerônimo da Serra (23 $43^{\prime} 37^{\prime \prime}$ S, 50 $53^{\circ} 07^{\prime \prime}$ W) and those of $C$. intermedium at Londrina ( $\left.23^{\circ} 27^{\prime} 21^{\prime \prime} \mathrm{S}, 51^{\circ} 1348^{\prime \prime} \mathrm{W}\right)$, both locations being in the southern Brazilian state of Paraná. Samples were cultivated at the Laboratory of Biodiversity and Ecosystem Restoration (LABRE) at the Universidade Estadual de Londrina, Paraná, Brazil. Vouchers were deposited at the FUEL herbarium. Cytogenetic analysis was carried out with young root tips that were pretreated with $0.05 \%(\mathrm{w} / \mathrm{v})$ aqueous colchicine (Sigma), fixed in 3:1(v:v) ethanol:acetic acid for $12 \mathrm{~h}$ to $24 \mathrm{~h}$ and stored at $-20^{\circ} \mathrm{C}$ until use. The samples were softened in a solution containing $4 \%$ $(\mathrm{w} / \mathrm{v})$ cellulase and $40 \%(\mathrm{w} / \mathrm{v})$ pectinase (all of Sigma) at $37^{\circ} \mathrm{C}$ for $1 \mathrm{~h}$ and then squashed in a drop of $45 \%$ acetic acid. The coverslips were subsequently removed in liquid nitrogen. Slides were used immediately after their preparation or then kept at $-20{ }^{\circ} \mathrm{C}$.

Genomic DNA of C. strigilatum was extracted from young leaves using the cetyltrimethyl ammonium bromide (CTAB) method described by Rogers and Bendich (1988). The GyRT1 (5' MRNATGTGYGTNGAYTAYMG) and GyRT4 (5' RCAYTTNSWNARYTTNGCR) degenerate primers (Friesen et al. 2001), giving a 450 bp product, were used for the PCR reactions. The band containing the $450 \mathrm{bp}$ fragment was purified with the Concert Rapid Gel Extraction System Kit (Gibco) and then re-amplified with the GyRT3 (5' YKNWSNGGNTAYCAYCARAT) and GyRT4 primers, as described above, to obtain a $320 \mathrm{bp}$ product. The last fragment was cloned in E. coli with the TOPO TA Cloning for Sequencing Kit (Invitrogen Life Technologies, USA) and several clones were sequenced using the DYEnamic ET dye Terminator Cycle Sequencing Kit (Amersham Pharmacia Biotech) on MEGABACE 1000 in the forward and reverse directions, according to the manufacturer's instructions. The sequence analysis and contig construction were performed with PhredPhrapConsed software (http://bozeman.mbt.washington.edu/consed/consed. html). The consensus sequence was compared with GeneBank sequences using the BLAST(X) Software tools. (http://www.ncbi.nlm.nih.gov).

The pCs 12 plasmid containing a segment of the Ty3/gypsy retroelement and the $\mathrm{p} T a 71$ plasmid containing 18S-5.8S-26S rDNA (Gerlach and Bedbrook, 1979) were used as probes. FISH was performed according to HeslopHarrison et al. (1991), with modifications. The 45S rDNA and Ty3/gypsy probes were labeled with biotin-14-dATP by nick translation (Invitrogen Bionick Kit). The slides were pretreated with RNase $(100 \mu \mathrm{g} / \mathrm{mL})$ at $37^{\circ} \mathrm{C}$ for $1 \mathrm{~h}$, washed in $2 \mathrm{xSSC}$, post-fixed in $4 \%$ paraformaldehyde $(\mathrm{w} / \mathrm{v})$, and washed again in $2 \mathrm{xSSC}$ at room temperature. Samples were dehydrated in a graded ethanol series and air-dried. Slides were treated with $30 \mu \mathrm{L}$ of a hybridization mixture composed of $100 \mathrm{ng}$ of labeled Ty3/gypsy probe, $50 \%(\mathrm{v} / \mathrm{v})$ formamide, $50 \%$ polyethylene glycol $(\mathrm{v} / \mathrm{v})$, 20xSSC, $100 \mathrm{ng}$ of fragmented E. coli DNA, and $10 \%(\mathrm{v} / \mathrm{v})$ SDS. The mixture was denatured at $70^{\circ} \mathrm{C}$ and chilled on ice prior to use. Both chromosomes and hybridization mixture were denatured/renatured using a thermal cycler at $90{ }^{\circ} \mathrm{C}$ for $10 \mathrm{~min}, 50^{\circ} \mathrm{C}$ for $10 \mathrm{~min}$ and $38^{\circ} \mathrm{C}$ for $10 \mathrm{~min}$. The hybridization was performed at $37^{\circ} \mathrm{C}$ overnight in a humidified chamber. The post-hybridization washes for the $\mathrm{p} C s 12$ probe were in $6 \mathrm{xSSC}$ for $20 \mathrm{~min}$ at room temperature, 6xSSC at $37{ }^{\circ} \mathrm{C}$ for $3 \mathrm{~min}$, and $4 \mathrm{xSSC} / 2 \%$ Tween 20 at room temperature for $5 \mathrm{~min}$ (about $40 \%$ of stringency to point out to dispersive pattern of Ty3/gypsy). Biotin-labeled probe was detected with avidin-fluorescein isothiocyanate (avidin-FITC) conjugate (Sigma). Chromosomes were analyzed without counterstaining, and photographed with Kodak Proimage 100 ISO film. Afterwards, slides were washed with $2 \mathrm{xSSC}$ and $4 \mathrm{xSSC}$ containing $0.2 \%$ Tween 20 $(\mathrm{v} / \mathrm{v})$ at room temperature for $30 \mathrm{~min}$ each, dehydrated in a graded ethanol series and air-dried. Slides were hybridized again with a $45 \mathrm{~S}$ rDNA probe, as described above, except for the blocking DNA that was calf thymus DNA (Invitrogen). Washes were performed in $2 \mathrm{xSSC}, 0.1 \mathrm{xSSC}$ containing $20 \%$ formamide, then in $0.1 \mathrm{xSSC}$ and finally in $2 \mathrm{xSSC}$ at $42{ }^{\circ} \mathrm{C}$ for $5 \mathrm{~min}$ each (about $80 \%$ of the stringency was due to the $45 \mathrm{~S}$ rDNA size and location pattern). The hybridization sites were detected again with avidin-FITC conjugate and the chromosomes counterstained with $2.5 \mu \mathrm{g} \mathrm{mL}^{-1}$ propidium iodide and photographed.

For characterization and differentiation of $C$. intermedium and C. strigilatum B-chromosomes, samples were submitted to $\mathrm{C}$-Giemsa and $\mathrm{C}-\mathrm{CMA}_{3} / \mathrm{DAPI}$ banding. Root tips were digested at $37{ }^{\circ} \mathrm{C}$ in a solution composed of $4 \%(\mathrm{w} / \mathrm{v})$ cellulase and $40 \%(\mathrm{w} / \mathrm{v})$ pectinase and the chromosome spread was made in a drop of $45 \%(\mathrm{v} / \mathrm{v})$ acetic acid. Coverslipped slides were frozen in liquid nitrogen and then the cover slips removed. Slides were aged for three days at room temperature, treated with $45 \%(\mathrm{v} / \mathrm{v})$ acetic acid, 5\% (w/v) barium hydroxide, and 2xSSC (pH 7) and then stained with $2 \%(\mathrm{w} / \mathrm{v})$ Giemsa (Schwarzacher et al., 
1980). Alternatively, the fixed slides were aged for three more days at room temperature and sequentially stained with $0.5 \mathrm{mg} \mathrm{mL}^{-1}$ chromomycin $\mathrm{A}_{3}\left(\mathrm{CMA}_{3}\right) \mathrm{GC}$-specific fluorochrome for $1.5 \mathrm{~h}$ and $2 \mu \mathrm{g} \mathrm{mL} \mathrm{m}^{-1} 4^{\text {'-6-diamidino-2- }}$ phenylindole (DAPI) AT-specific fluorochrome for $30 \mathrm{~min}$ (Vanzela et al., 2002). Samples stained with Giemsa were mounted with Entellan (Merck), but those stained with the fluorochromes were mounted in a medium composed of glycerol/Mcllvaine buffer ( $\mathrm{pH}$ 7) containing $2.5 \mathrm{mM}$ $\mathrm{MgCl}_{2}$. The cells were photographed with Imagelink HQ ASA 25 or T-max ASA 100 film (Kodak). Analyses were based on five well-spread metaphases for each treatment.

\section{Results}

The reaction using the GyRT1/GyRT4 primers generated several fragments of different sizes, including a stronger band of about $450 \mathrm{bp}$ (Figure 1A). This fragment was isolated, purified and used as in Friesen et al. (2001) and then re-isolated, purified and used as a template for a new reaction with the GyRT3 (internal) and GyRT4 primers. This new PCR reaction produced a band of about $320 \mathrm{bp}$ (Figure 1B), which was isolated, purified and cloned. Clones were sequenced and one corresponding to the Ty3/gypsy elements (pCs12) included in the NCBI GeneBank Sequence Viewer.htm (gi:53801553, accession number AY744673). The sequence showed similarity with several related reverse transcriptase fragments (Figure 2A). The comparisons showed that the $\mathrm{pCs} 12$ insert was very similar to sequences of Ty3/gypsy-like retroelements found in monocotyledons and dicotyledons. The strongest similarity was with a retroelement of the monocotyledon Oryza sativa (rice) (gi: 37533358, e-value 2e-29) followed by the Solanum demissum (gi: 47824972, e-value 2e-29) and Elaeis guineensis (gi:22859203, e-value 2e-28) and the monocotyledon Ananas comosus (gi:2995405, e-value 2e-28), and was also similar to the monocotyledon Lolium multiflorum (gi:13559324, e-value 2e-25). Figure 2B shows the more significant alignment obtained with BLAST(x).

The FISH analysis using the $\mathrm{pCs} 12$ probe revealed diffuse signals along the chromosomes of $C$. strigilatum,

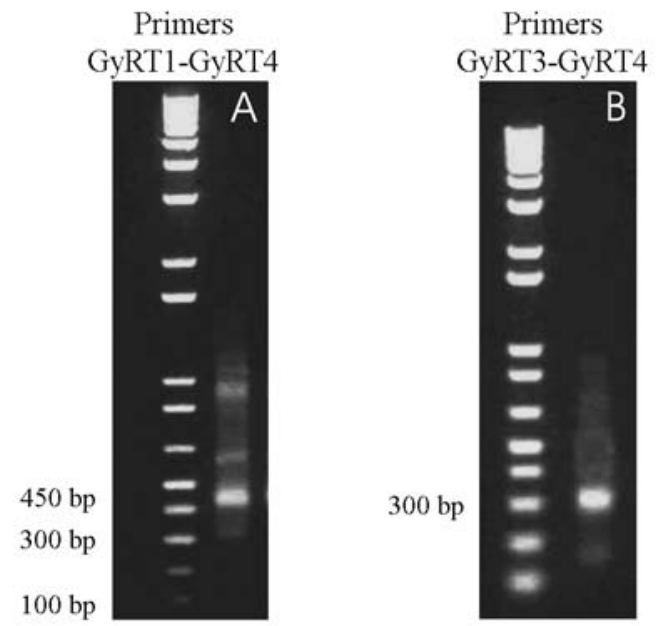

Figure 1 - A) Results of the PCR reaction using the GyRT1/GyRT4 primers. The 450 bp band was removed, purified and used in a semi-nested PCR reaction. B) Results of semi-nested PCR reaction using the GyRT3/GyRT4 primers. The 320 bp band was purified, cloned and several clones sequenced. The $\mathrm{p} C s 12$ probe was labeled and used for fluorescence in situ hybridization (FISH).

but brighter signals were visualized at the terminal positions of a metacentric pair and on the short arm of the eighth chromosome pair. Besides the signals visualized on the A complement chromosomes, two more accentuated signals were detected at intercalary positions on the short and long arms of a B chromosome (Figure 3A and 3I). A negative band was detected in the intercalary region of the short arm of a C. strigilatum chromosome pair showing the absence of hybridization with $\mathrm{p} C s 12$ probe (see arrow heads in Figure $3 \mathrm{~A}$ ). The absence hybridization signals on these chromosomal segments indicated that the low stringency used (about $40 \%$ ) did not interfered nor did it overestimate the dispersive pattern of occurrence of Ty3/gypsy elements (pCs12). In C. intermedium FISH also revealed dispersed signals along the chromosomes, very similar to that observed in the A complement of $C$. strigilatum, and also the small hybridization signals at the terminal positions of a metacentric pair and on the short arm of the eighth chromosome pair as well (Figure 3B). The two C. intermedium

\section{GCATTTTCTTAGTTTGGCGACGAGTTTGTGCTCCTTGAGTGTCTGTAAGG TGATCCTGCGGTGTTCCACACGGTCTCCССTACTTTTTGAGTACAACAGT ATATCATCAATAAGTACAATAATCAAGGTGTCCAAATAAGGCCTGAACAC TCGATTCATTAAATCCATGAAAGCTGCAGGGGCATTTGTTAGCTCGAAAG GCGATTACCAAAAATTCATAGTGCCCGTATCTAGTCCTGAACACTGTTTT TAGGAAGTTCCCCCCСTAATCGTCATTTGATGATAACCTCTGCA}

Figure 2a - Results of sequencing of the $\mathrm{p} C s 12$ probe obtained by PCR from Cestrum strigilatum.

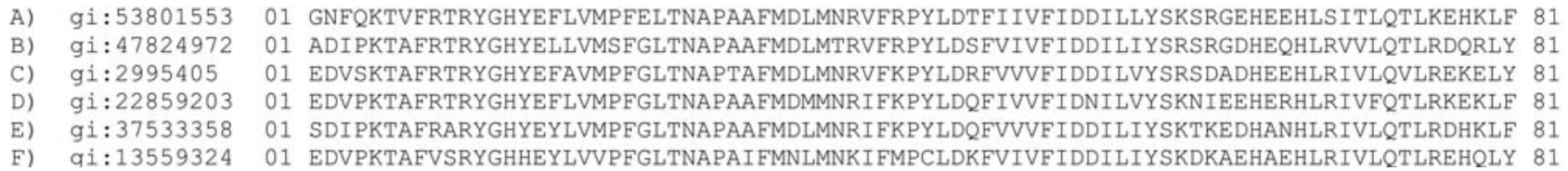

Figure 2b - ClustalW alignment comparing the amino acid sequence of the Cestrum strigilatum retroelement with the most similar sequences recovered from databanks. A) C. strigilatum, B) Solanum demissum, C) Ananas comosus, D) Elaeis guineensis, E) Oryza sativa, F) Lolium multiflorum. 
B-chromosomes also showed hybridization signals on both arms, but both were larger and more evident than the signals obtained on the $C$. strigilatum B-chromosome (Figure $3 \mathrm{~N})$. The accentuated $\mathrm{pCs} 12$ hybridization signals observed in B-chromosomes also reinforce the fact that the $40 \%$ stringency did not overestimate the dispersive occurrence of Ty3-gypsy on the A-chromosomes of both species. The slides hybridized with $\mathrm{p} C s 12$ probe were washed and again hybridized with the $45 \mathrm{~S}$ rDNA probe. In both species, bright terminal rDNA signals were visualized on the same positions as the pCs 12 probe signals (Figure 3C and 3D). However, in $C$. intermedium the bright intensity was not as strong as on the terminal region of the A-chromosomes. It is important to point out that, unfortunately, not all the eight 45S rDNA sites were detected after the new hybridization, probably due to technical problems. However, based on the physical map proposed by Fregonezi et al. (2006), it is safe to say that at least one representative of each chromosome pair containing a NOR was detected by the $\mathrm{p} T a 71$ probe. The chromosome region observed as a negative segment after the hybridization with the $\mathrm{pCs} 12$ probe (see arrows at Figures $3 \mathrm{~A}$ and $3 \mathrm{~B}$ ) appeared stained with propidium iodide (Figures 3C and 3D). This further shows that the stringency and hybridization technique was correct. The Figure $3 \mathrm{E}$ to $3 \mathrm{I}$ (Bs of C. strigilatum) and $3 \mathrm{~J}$ to $3 \mathrm{~N}$ (B-chromosomes of $C$. intermedium) show a comparison of B-chro-

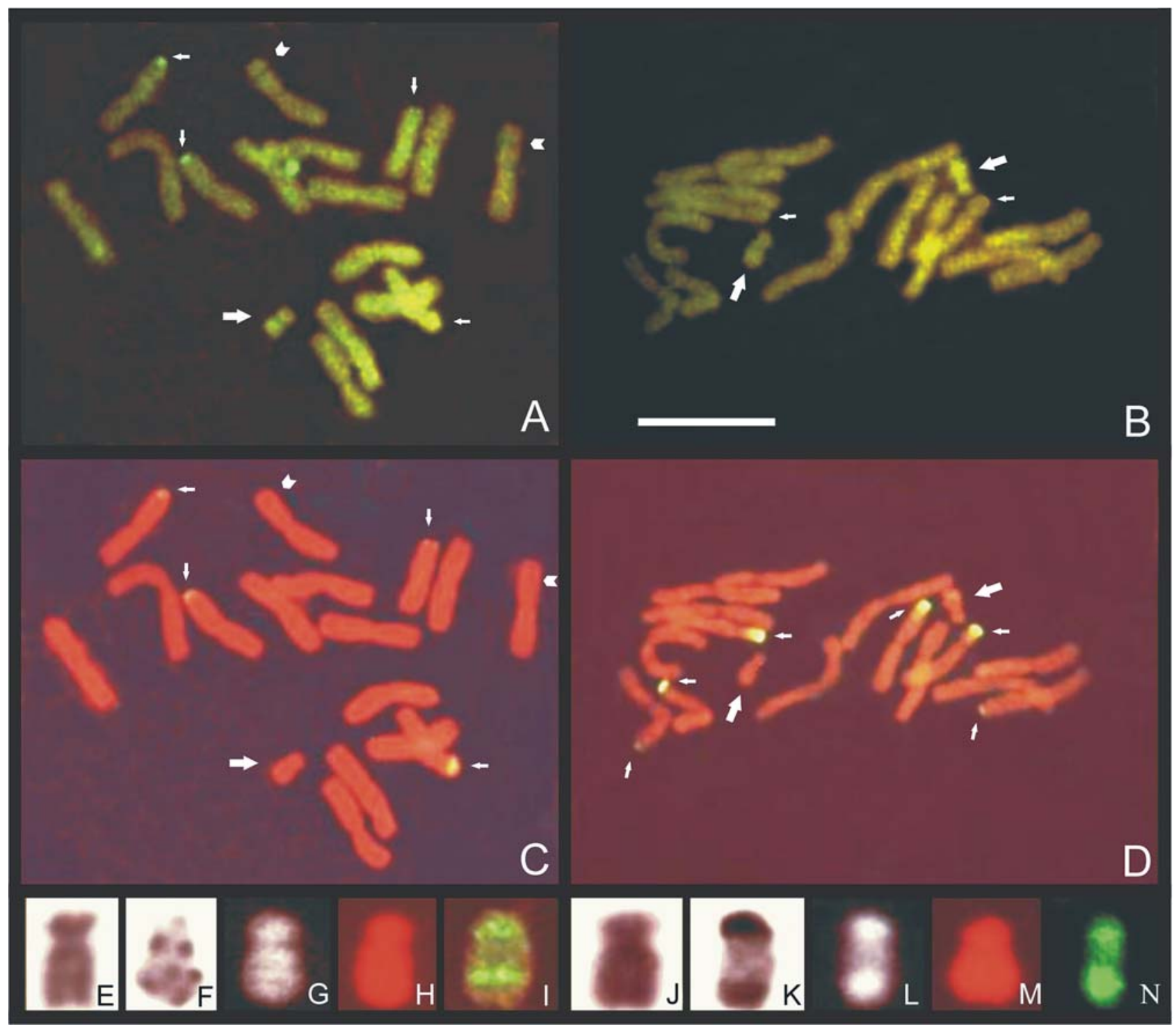

Figure 3 - A) Fluorescence in situ hybridization (FISH) with the pCs 12 probe in Cestrum strigilatum. B) FISH with pCs 12 probe in Cestrum intermedium. Small arrows point out terminal sites and the large arrow point out B-chromosomes. C) FISH with pTa71 probe in C. strigilatum. D) FISH with $\mathrm{p} T a 71$ probe in $C$. intermedium. Small arrows point out the terminal $45 \mathrm{~S}$ rDNA sites and the large arrow point out B-chromosomes. The arrow heads show that the chromosome region observable as a negative segment after the hybridization with the $\mathrm{p} C s 12$ probe $(3 \mathrm{~A})$ appears perfectly stained with propidium iodide (3B). E-I) B-chromosomes of C. strigilatum, treated for conventional Giemsa, Giemsa C-banding, DAPI, propidium iodide and FISH with $\mathrm{p} C s 12$ probe, respectively. J-N) B-chromosomes of $C$. intermedium, treated for conventional Giemsa, Giemsa C-banding, DAPI, propidium iodide and FISH with the $\mathrm{pCS} 12$ probe, respectively. Bar, $10 \mu \mathrm{m}$. 
mosomes analyzed by several techniques, that is, conventional Giemsa staining (3E and J), C-Giemsa (3F and 3K), C-DAPI ( $3 \mathrm{G}$ and $3 \mathrm{~L}$ ), propidium iodide $(3 \mathrm{H}$ and $3 \mathrm{M})$, and FISH with pCs 12 (3I and $3 \mathrm{~N}$ ). These techniques showed that B-chromosomes are very similar when stained with Giemsa and propidium iodide, but exhibit differences evident when treated with C-Giemsa and C-DAPI banding and FISH with the $\mathrm{pCs} 12$ probe.

\section{Discussion}

The retroelement families are characterized by heterogeneity in many of their sequences, mainly due to the propagation mechanism through reverse transcriptase, which produces base substitutions in the copy processes (Van Sluys et al., 2001). The Ty1/copia and Ty3/gypsy retroelement groups usually occur in multiple families in related organisms, but different retroelement families can exist within the genome of an organism. Marín and Llórens (2000) found nine different Ty3/gypsy forms in Arabidopsis thaliana through the comparison of conserved sequences. It was also suggested by these authors that the differences among these forms might have originated prior to the separation of monocotyledons and dicotyledons. The occurrence of different retrotransposon forms could be considered a general feature of the modern angiosperms. The sequence found in Cestrum was highly similar to the retroelements of three monocotyledons (O. sativa, A. comosum and L. multiflorum) and two dicotyledons (S. demissum and E. guineensis) and if these data were analyzed from only the point of view of the Ty3/gypsy retroelements, the possible ancestral genome of the dicotyledons could contain Ty3/gypsy retroelements similar to the segment cloned in $\mathrm{p} C s 12$.

In regard to the physical position of the retroelements, these can occur dispersed along the chromosomes, or concentrated in specific regions, according to the genomic organization of the species (Heslop-Harrison et al., 1997). In our study, C. strigilatum and C. intermedium showed a diffuse distribution of the $\mathrm{pCs} 12$ probe, indicating that the Ty3-gypsy retroelements also occur distributed along the chromosomes. Besides this general dispersed tendency, both species exhibited a concentration of these elements in some terminal regions, associated with NORs, which are also associated with GC-rich heterochromatin (Fregonezi et al., 2004). Retroelements have previously been reported to be associated with the NORs of some Triticeae (Belyayev et al., 2001), with the heterochromatin of Allium cepa (onion) (Pearce et al., 1996) and A. thaliana (Brandes et al., 1997), and with centromeric regions in several monocotyledons (Kumar and Bennetzen, 1999) and dicotyledons (Santini et al., 2002). Retrotransposons have also been reported to be associated with the whole genome as segments dispersed along the A- and D-chromosomes of Avena sativa (oat) (Linares et al., 2001). However, the association of the retroelements with specific DNA segments is not obligatory. Schmidt et al. (1995) demonstrated that the
BNR1 and Tbv retrotransposon families of Beta vulgaris (sugar beet) are heterogeneous and highly amplified on the genome but are largely excluded from regions rich in 18S5.8S-25S rRNA genes.

Hybridization signals showed that the B-chromosomes of both $C$. strigilatum and $C$. intermedium possess Ty3-gypsy elements but with visible differences in the number, position, and size of the repeats. This is in agreement with the suggestion of Camacho et al. (2000) for B-chromosomes. According to Jones and Houben (2003) B-chromosomes are described as heterochromatic in about half of plants that carry them. In general, the repetitive DNA families of Bs are similar to that of their A chromosomes. Species of Cestrum contain more than one family of repetitive DNA, which seems indicate different dynamics from that observed in the A-chromosomes for C-banding and C-DAPI segments (Fregonezi et al., 2004) and Ty3-gypsy elements. Repetitive DNA families occupying a large portion of B-chromosomes have already been described in several plants species, such as Brachycome dichromosomatica (Houben et al., 2001), Medicago (Hossain and Bauchan, 1999) and Zea mays (Stark et al., 1996). The presence of Ty3-gypsy retroelements in the B-chromosomes of C. strigilatum and C. intermedium indicates that these repetitive segments may contribute to the formation and stabilization of B-chromosomes in these species, and that this might have occurred through the amplification of existing segments in the chromosomes of the normal complement.

It is important to point out that the probe used by us was obtained with degenerate primers designed for the conserved portion of the reverse transcriptase of the gypsy elements, and that the reverse transcriptase genes of all retrotransposons are related by their monophyletic origin (Hansen and Heslop-Harrison, 2004). As different retroelements can also have followed different evolutionary pathways it is not uncommon to find associations among different types of retrotransposons and different DNA families, as those found by us among the Ty3/gypsy retroelements, NOR sites and B-chromosomes of $C$. strigilatum and $C$. intermedium.

\section{Acknowledgments}

This study was supported by the Brazilian agencies CAPES, IAP-SEMA, Fundação Araucária and by ProPPG-UEL. The authors thank the Brazilian agency CNPq for a fellowship, and also to José Marcelo Domingues Torezan for constructive criticism and Dr. A. Leyva for reading the manuscript.

\section{References}

Belyayev A, Raskina O and Nevo E (2001) Chromosomal distribution of reverse transcriptase-containing retroelements in two Triticeae species. Chromosome Res 9:129-136. 
Berg C and Greilhuber J (1992) Cold-sensitive chromosome regions and their relation to constitutive heterochromatin in Cestrum parqui (Solanaceae). Genome 35:921-930.

Berg C and Greilhuber J (1993a) Cold-sensitive chromosome regions and heterochromatin in Cestrum (Solanaceae): $C$. strigilatum, C. fasciculatum, and C. elegans. Plant Syst Evol 185:133-151.

Berg C and Greilhuber J (1993b) Cold-sensitive regions and heterochromatin in Cestrum aurantiacum (Solanaceae). Plant Syst Evol 185:259-273.

Brandes A, Heslop-Harrison JS, Kamm A, Kubis S, Doudrick RL and Schmidt T (1997) Comparative analysis of the chromosomal and genomic organization of Ty1-copia-like retrotransposons in pteridophytes, gymnosperms and angiosperms. Plant Mol Biol 33:11-21.

Camacho JPM, Sharbel TF and Beukeboom LW (2000) B chromosome evolution. Phil Trans R Soc Lond B. 355:163-178.

D'Arcy WG (1979) The classification of the Solanaceae. In: Hawkes JG, Lester RN and Skelding AD (eds) The Biology and Taxonomy of the Solanaceae. Academic Press, London, $738 \mathrm{pp}$.

Fregonezi JN, Rocha C, Torezan JMD and Vanzela ALL (2004) The occurrence of different Bs in Cestrum intermedium and C. strigilatum (Solanaceae) evidenced by chromosome banding. Cytogenet Genome Res 106:184-188.

Fregonezi JN, Fernandes T, Torezan JMD, Vieira AOS and Vanzela ALL (2006) Karyotype differentiation of four Cestrum species (Solanaceae) based on the physical mapping of repetitive DNA. Genet Mol Biol 29:97-104.

Friesen N, Brandes A and Heslop-Harrison JS (2001) Diversity, origin, and distribution of retrotransposons (gypsy and copia) in conifers. Mol Biol Evol 18:1176-1188.

Gerlach WL and Bedbrook JR (1979) Cloning and characterization of ribossomal RNA genes from wheat and barley. Nucl Acid Res 7:1869-1885.

Hansen CN and Heslop-Harrison JS (2004) Sequences and phylogenies of plant pararetroviruses, viruses and transposable elements. Adv Bot Res 41:165-193.

Heslop-Harrison JS, Schwarzacher T, Anamthewat-Jonsson K, Leitch AR, Shi M and Leitch IJ (1991) In situ hybridization with automated chromosome denaturation. Technique 3:106-109.

Heslop-Harrison JS, Brandes A, Taketa S, Schmidt T, Vershinin AV, Alkhimova EG, Kamm A, Kamm A, Doudrick RL, Schwarzacher T, et al. (1997) The chromosomal distributions of Ty1-copia group retrotransposable elements in higher plants and their implications for genome evolution. Genetica 100:197-204.

Houben A, Verlin D, Leach CR and Timmis JN (2001) The genomic complexity of $\mathrm{B}$ chromosomes of Brachycome dichromosomatica. Chromosoma 110:451-459.
Hossain MA and Bauchan GR (1999) Identification of B chromosomes using Giemsa banding in Medicago. J Hered 90:428429.

Jones N and Houben A (2003) B chromosomes in plants: Escapees from the A chromosome genome? Trend Plant Sci $8: 417-423$.

Kumar A and Benntzen JL (1999) Plant retrotransposons. Annu Rev Genet 33:479-532.

L'Homme Y, Seguin A and Tremblay FM (2000) Different classes of retrotransposons in coniferous spruce species. Genome 43:1084-1089.

Linares C, Loarce Y, Serna A and Fominaya A (2001) Isolation and characterization of two novel retrotransposons of the Ty1-copia group in oat genomes. Chromosoma 110:115123.

Marín I and Lloréns C (2000) Ty3/Gypsy Retrotransposons: Description of new Arabidopsis thaliana elements and evolutionary perspectives from comparative genomic data. Mol Biol Evol 17:1040-1049.

Pearce SR, Pich U, Harrison G, Flavell AJ, Heslop-Harrison JS, Schubert I and Kumar A (1996) The Ty1-copia group retrotransposons, a major component of Allium cepa genome, are distributed throughout the chromosomes but are enriched in the terminal heterochromatin. Chromosome Res 4:357-364.

Rogers SO and Bendich AJ (1988) Extraction of DNA from milligram amounts of fresh, herbarium, and mummified plant tissues. Plant Mol Biol 5:69-76.

Santini S, Cavallini A, Natali L, Minelli S, Maggini F and Cionini PG (2002) Ty1/copia- and Ty3/Gypsy-like DNA sequences in Helianthus species. Chromosoma 111:192-200.

Schmidt T, Kubis S and Heslop-Harrison JS (1995) Analysis and chromosomal localization of retrotransposons in sugar beet (Beta vulgaris L.): LINEs and Ty1-copia-like elements as major components of the genome. Chromosome Res 3:335345 .

Schwarzacher TP, Ambros P and Schweizer D (1980) Application of Giemsa banding to orchid karyotype analysis. Plant Syst Evol 134:293-297.

Stark EA, Connerton I, Bennett ST, Barnes SR, Parker JS and Forster JW (1996) Molecular analysis of the structure of the maize B chromosome. Chromosome Res 4:15-23.

Van Sluys M, Scortecci KC and Costa APP (2001) O genoma instável, seqüências genéticas móveis. In: Matioli SR (ed) Biologia Molecular e Evolução. Holos Editora, Ribeirão Preto, pp 70-81.

Vanzela ALL, Ruas CF, Oliveira MF and Ruas PM (2002) Characterization of diploid, tetraploid and hexaploid Helianthus species by chromosome banding and FISH with $45 \mathrm{~S}$ rDNA probe. Genetica 114:105-111.

Associate Editor: Marcelo Guerra 